\title{
Comparison between Digital Fresnel Holography and Digital Image-Plane Holography: The Role of the Imaging Aperture
}

\author{
M. Karray $\cdot$ P. Slangen $\cdot$ P. Picart
}

\begin{abstract}
Optical techniques are now broadly used in the field of experimental mechanics. The main advantages are they are non intrusive and no contact. Moreover optical techniques lead to full spatial resolution displacement maps enabling the computing of mechanical value also in high spatial resolution. For mesoscopic measurements, digital image correlation can be used. Digital holographic interferometry is well suited for quantitative measurement of very small displacement maps on the microscopic scale. This paper presents a detailed analysis so as to compare digital Fresnel holography and digital image-plane holography. The analysis is based on both theoretical and
\end{abstract}

\author{
M. Karray $\cdot$ P. Picart \\ LAUM CNRS, Université du Maine, \\ Avenue Olivier Messiaen, \\ 72085 LE MANS Cedex 9, France \\ M. Karray \\ e-mail: mayssa.karray@univ-lemans.fr \\ P. Picart \\ e-mail: pascal.picart@univ-lemans.fr \\ P. Slangen $(\bowtie)$ \\ Ecole des Mines d'Alès, \\ 6 Avenue de Clavière, \\ 30100 ALES, France \\ e-mail: pierre.slangen@mines-ales.fr \\ P. Picart \\ ENSIM, École Nationale Supérieure d'Ingénieurs du Mans, \\ rue Aristote, \\ 72085 LE MANS Cedex 9, France
}

experimental analysis. Particularly, a theoretical analysis of the influence of the aperture and lens in the case of image-plane holography is proposed. Optimal filtering and image recovering conditions are thus established. Experimental results show the appropriateness of the theoretical analysis.

Keywords Digital holography. Phase measurement . Displacement measurement - Deformation measurement . Imaging aperture

\section{Introduction}

Digital holography was experimentally established in the 90's [1, 2]. Lately, many fascinating possibilities have been demonstrated: focusing can be chosen freely [3], a single hologram can provide amplitude-contrast and phase-contrast microscopic imaging [4], image aberrations can be compensated [5], properties of materials can be investigated [6], digital color holography [7, 8] and time-averaging are also possible [9]. Theory and reconstruction algorithms for digital holography have been described by several authors [10-14]. The processing of digital holograms is generally based on the discrete Fresnel transform [11], which is applied on a single digital hologram [1] or after a pre-processing based on phase shifting $[2,15]$.

Digital holography exhibits various architectures such as Fresnel holography (DFH), Fourier holography, Lens-less Fourier holography and image-plane holography (DIPH) [11]. Particularly, holographic techniques give a fruitful 
contribution to the analysis of mechanical structures under strain, by providing whole field information on displacement $[8,11,13,16]$.

The methods of DFH and DIPH find their interest in contact-less metrology with applications in mechanical strain, vibrations, displacement field or surface shape measurements. There are some strong similarities between both methods, especially concerning data processing. However, some figures of merit explaining the advantages and the drawbacks of the methods have not been discussed in literature. Compared to Fourier and Fresnel holography, the image-plane configuration shows some particularities that are detailed in this paper: the role of the aperture diaphragm of the imaging system. This paper proposes an analysis of the influence of the aperture on the basis of four criteria: filtering and numerical processing, spatial resolution and decorrelation noise. "Theoretical Basics" presents the basic fundamentals. "Figures of Merit" describes the figures of merit. Experimental results are summarized in "Experimental Results". "Conclusion" draws some conclusions about the study.

As discussed in the previous section, processing of digital holograms can be based on phase-shifting [2], requiring at least three recordings to efficiently process the data [15]. Note that a huge amount of literature describing phase shifting arrangements and processing aspects is available, and will be not discussed here. This paper focuses on the case where off-axis digital holograms are recorded $[1,11$, 14]. This choice is justified as follows: recording a single hologram per instant is a powerful tool to study dynamic events and to carry out high speed acquisition. Examples demonstrating the potentiality of such an approach can be found in [17] for the DFH method and [18, 19] for the DIPH one. As we aim at comparing objectively both methods, the same constraints must be applied. Indeed, the experimental optimization of such methods can be performed according to several degrees of freedom. The amplitude of the reference wave can particularly be increased, compared to that of the object beam, in order to get more flexibility in the Shannon conditions when recording, especially as concerns the non-overlapping of the three diffraction orders [20, 21]. Here, we consider that the reference waves of both methods are plane waves and are experimentally adjusted to have the same amplitude. Focus is on the information carried by the object wave when the object is illuminated under the same conditions. The spatial frequencies of the reference beam are fixed and for DIPH, a lens is added to form the image onto the sensor area. This lens is associated to an iris diaphragm, whose role is to limit the aperture of the beam passing through the imaging system. In 1997 [20], G. Pedrini presented the first comparative study between DFH and DIPH and he pointed out that DIPH is a particular case of
DFH because the digital reconstructed hologram leads to the simulation of the complex amplitude in the space. G. Pedrini [20] discussed qualitatively on the object reconstructions and the spatial resolutions of both methods. The approach proposed here aims at taking into account both theoretical and experimental aspects to achieve an objective comparison.

Note that the DIPH configuration also corresponds to a particular set-up of the speckle interferometry method discussed in the paper of P. Jacquot [22]. However, to avoid any confusion, the goal of the paper is not to establish a generalized comparison between digital holography and speckle interferometry. Indeed, speckle interferometry systems are overabundant and consist of at least three principal families: the in-line reference, the double illumination and the shearing configurations [22]. In the in-line reference family, that appears to have some similarity with DIPH, several variants may be considered including for example the choice of a speckle or a smooth reference beam, and a strict or relaxed in-line alignment with the object beam, each presenting advantages and drawbacks of their own.

\section{Theoretical Basics}

This section presents the theoretical background of both DFH and DIPH methods by considering the recording/processing of a unique digitally recorded hologram. As a general rule, let us consider an extended object, sized $\Delta A_{x} \times \Delta A_{y}$, illuminated by a coherent monochromatic wave with wavelength $\lambda$ and a set of reference coordinates attached to the object $(X, Y, z)$ and to the recording plane $(x, y, z)$. In the paper, we consider a recording sensor $M \times N$ pixels with pitches $p_{x}=p_{y}$. To differentiate both methods, the digital hologram will be called a "Fresnelgram" for the Fresnel configuration whereas it will be called an "imagegram" for the imageplane one.

Digital Fresnel Holography: Recording and Reconstruction

In the case of digital Fresnel holography, the object diffracts a wave to the recording plane, localized at distance $d_{0}$. Figure 1 illustrates the experimental setup and notations.

The object surface generates a wave front that will be noted according to equation (1):

$A(X, Y)=A_{0}(X, Y) \exp \left[i \psi_{0}(X, Y)\right]$. 
Fig. 1 Optical setup for DFH

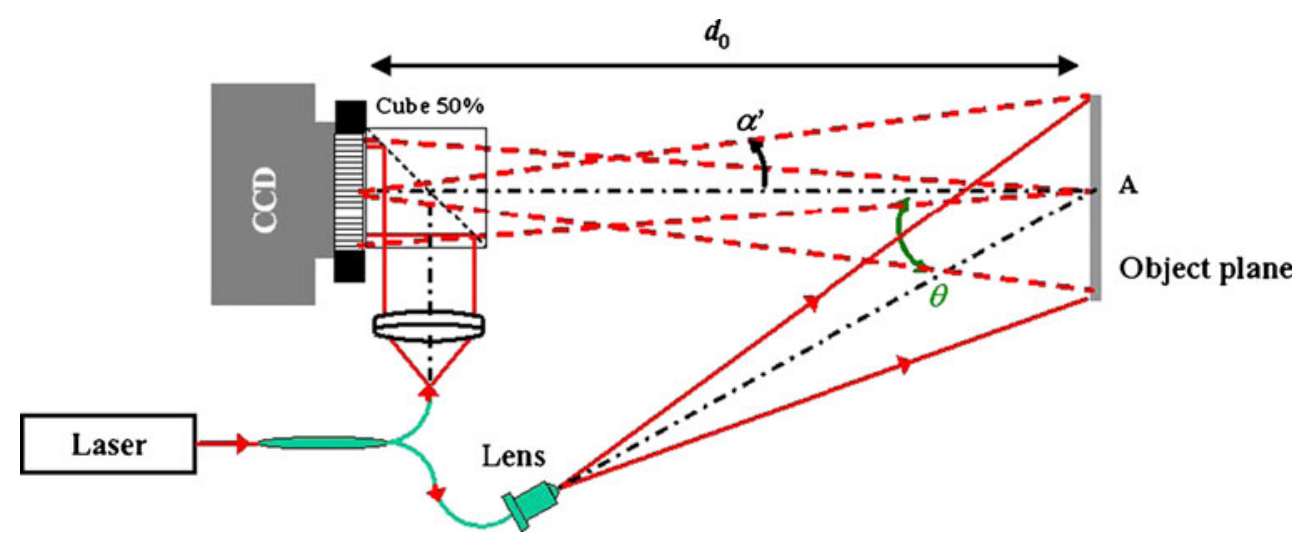

Amplitude $A_{0}$ describes the object reflectivity and phase $\psi_{0}$ describes its surface or shape $(i=\sqrt{-1})$. Phase $\psi_{0}$ is random and uniformly distributed over the range $]-\pi,+\pi]$.
When taking into account the diffraction theory under the Fresnel approximations [23], the object wave diffracted at distance $d_{0}$ is expressed by the following relation:

$$
\begin{aligned}
O\left(x, y, d_{0}\right)= & -\frac{i \exp \left(2 i \pi d_{0} / \lambda\right)}{\lambda d_{0}} \exp \left(\frac{i \pi}{\lambda d_{0}}\left(x^{2}+y^{2}\right)\right) \\
& \times \iint A(X, Y) \exp \left(\frac{i \pi}{\lambda d_{0}}\left(X^{2}+Y^{2}\right)\right) \exp \left(-\frac{2 i \pi}{\lambda d_{0}}(x X+y Y)\right) d X d Y .
\end{aligned}
$$

Note that since the object is rough, the diffracted field at distance $d_{0}$ is a speckle field which has a random and uniform phase over the range] $-\pi,+\pi]$. In the $2 \mathrm{D}$ Fourier space, the object wave occupies a spatial frequency bandwidth equal to $(\Delta u \times \Delta v)=\left(\Delta A_{x} / \lambda d_{0} \times \Delta A_{y} / \lambda d_{0}\right)$. In the recording plane, the object wave is mixed with a plane reference wave written as:

$R(X, Y)=a_{r} \exp \left(2 i \pi\left(u_{0} X+v_{0} Y\right)\right)$,

with $a_{r}$ the modulus and $\left(u_{0}, v_{0}\right)$ the carrier spatial frequencies. When $\left(u_{0}, v_{0}\right) \neq(0,0)$ we get "off-axis digital holography" while when $\left(u_{0}, v_{0}\right)=(0,0)$, we get "in-line digital holography". As pointed out, we consider here the case of "off-axis digital holography". The total intensity received by the recording sensor is the Fresnelgram, written as:

$H=|O|^{2}+|R|^{2}+O R^{*}+O^{*} R$.

The Shannon theorem applied to off-axis DFH, resulting in the spatial separation of the three diffraction orders appearing in equation (4), leads to the optimal recording distance [14]. It is given for a circular object shape with diameter $\Delta A=\Delta A_{x}=\Delta A_{y}$ :

$d_{0}=\frac{(2+3 \sqrt{2}) p_{x}}{2 \lambda} \Delta A$.

Ideally, the spatial frequencies of the reference wave must be adjusted to $\left(u_{0}, v_{0}\right)=\left( \pm(1 / 2-1 /(2+3 \sqrt{ } 2)) / p_{x}, \pm(1 / 2-\right.$ $\left.1 /(2+3 \sqrt{2})) / p_{y}\right)$ for the circular object [14]. Practically, the spatial frequencies can be adjusted following this method: the reference beam is perpendicular to the recording plane but the object is laterally shifted by quantities:

$$
\left\{\begin{array}{l}
\Delta X=\frac{\lambda d_{0}}{p_{x}}\left(\frac{1}{2}-\frac{1}{2+3 \sqrt{2}}\right) \\
\Delta Y=\frac{\lambda d_{0}}{p_{y}}\left(\frac{1}{2}-\frac{1}{2+3 \sqrt{2}}\right)
\end{array} .\right.
$$

The reconstructions of the amplitude and the phase of the encoded object are based on the numerical simulation of light diffraction on the numerical aperture included in the digital hologram. For a reconstruction distance equal to $d_{r}=-d_{0}$, the reconstructed field $A_{r}$ is given by the discrete version of equation (2) (known as S-FFT algorithm, or also DFT: discrete Fresnel transform) $[1,11,14]$. If the reconstructed plane is computed with $(K, L) \geq(M, N)$ data points, then the sampling pitches in the reconstructed plane are equal to $\Delta \eta=\lambda d_{0} / L p_{x}$ and $\Delta \xi=\lambda d_{0} / K p_{y}[11,14]$. The reconstructed field is given by the following relation, the unnecessary factors and phase terms being removed,

$$
\begin{aligned}
A_{r}(n \Delta \eta, m \Delta \xi)= & \sum_{k=0}^{k=K-1} \sum_{l=0}^{l=L-1} H\left(l p_{x}, k p_{y}\right) \exp \left[-\frac{i \pi}{\lambda d_{0}}\left(l^{2} p_{x}^{2}+k^{2} p_{y}^{2}\right)\right] \\
& \exp \left[2 i \pi\left(\frac{\ln }{L}+\frac{k m}{K}\right)\right]
\end{aligned}
$$

where $l, k, n, m$ are indices corresponding to discrete versions of respectively $X, Y, x, y$. The +1 order is then localized at 
spatial coordinates $\left(\lambda d_{0} u_{0}, \lambda d_{0} v_{0}\right)$. Due to Shannon conditions, the minimum distance that can be put in the algorithm is given by $d_{0} \geq \max \left\{N p_{x}{ }^{2} / \lambda, M p_{y}{ }^{2} / \lambda\right\}$. The computation leads to complex-valued results, from which the amplitude image (modulus) and the phase image (argument) can be extracted. The discrete Fresnel transform is adapted to a large range of object sizes and shapes.

The second possibility to reconstruct the object from the Fresnelgram is based on the convolution formulae of diffraction. An exhaustive description was provided by Kreis in 1997 [11] and adjustable magnification was described in [12, 24-26]. The reconstructed field is obtained by this convolution equation ( $\otimes$ means convolution), at distance $d_{r}$ :

$A_{r}\left(x, y, d_{r}\right)=\{w(x, y) H(x, y)\} \otimes h\left(x, y, d_{r}\right)$.

where $h\left(x, y, d_{r}\right)$ is the kernel associated to diffraction along distance $d_{r}, w(x, y)=\exp \left(i \pi\left(x^{2}+y^{2}\right) / \lambda R_{c}\right)$ is a numerical spherical wave front having a curvature radius $R_{c}$. The reconstruction parameters are linked by the magnification of the reconstructed image, $\gamma$, such that $d_{r}=-\gamma d_{0}, R_{c}=\gamma d_{0} /(\gamma$ -1). The magnification can be chosen according to $\gamma=$ $\min \left\{L p_{x} / \Delta A_{x}, K p_{y} / \Delta A_{y}\right\}$, meaning that the reconstructed object will fully lie in the reconstructed horizon sized $L p_{x} \times K p_{y}$ [12]. The convolution kernel can be the impulse response of the free space propagation. Such a kernel leads to a transfer function, which is the Fourier transform of the impulse response. The mathematical expression of the kernel is given by Goodman [23] and must be adapted to off-axis holography [24]:

$$
\begin{aligned}
h\left(x, y, d_{r}\right)= & \frac{i d_{r}}{\lambda} \frac{\exp \left[2 i \pi / \lambda \sqrt{d_{r}^{2}+x^{2}+y^{2}}\right]}{d_{r}^{2}+x^{2}+y^{2}} \\
& \times \exp \left(-2 i \pi\left(u_{0} x+v_{0} y\right)\right),
\end{aligned}
$$

The angular spectrum transfer function can also be used as the transfer function of the reconstruction process. In this case the mathematical expression has to be adapted [12] and given by:

$G\left(u, v, d_{r}\right)=\left\{\begin{array}{c}\exp \left[2 i \pi d_{r} / \lambda \sqrt{1-\lambda^{2}\left(u-u_{0}\right)^{2}-\lambda^{2}\left(v-v_{0}\right)^{2}}\right] \\ \quad \text { if }\left|u-u_{0}\right| \leq L p_{x} / 2 \lambda d_{r} \text { and }\left|v-v_{0}\right| \leq K p_{y} / 2 \lambda d_{r}, \\ 0 \quad \text { elsewhere }\end{array}\right.$

The reader may look at references $[12,24,25]$ for further details regarding the reconstruction process. The practical computation of such an equation can be performed according to the properties of the Fourier transform, thus leading to double Fourier transform algorithm (D-FFT):

$A_{r}=F T^{-1}[F T[w H] \times F T[h]]$, which includes three FFT's, if using the impulse response, while only two FFT's, when using the angular spectrum transfer function:

$A_{r}=F T^{-1}[F T[w H] \times G]$,

In the D-FFT algorithms the reconstructed object is sampled by a number of data points that can be chosen freely with $(K, L) \geq(M, N)$, whereas with the S-FFT algorithm, the number of useful data points sampling the reconstructed object is given by the ratio $\left(\Delta A_{x} / \Delta \eta ; \Delta A_{y} / \Delta \xi\right)$.

Digital Image-Plane Holography: Recording and Reconstruction

In the case of DIPH, an imaging lens is associated to a variable aperture close to the lens. In the method proposed in $[20,27]$, the aperture is placed at the front focal plane of the lens. In this study, we consider the case of commercial lenses for which the aperture is not localized at the focal plane. The aperture has a diameter $\phi_{D}$ and is placed at distance $d_{D}$ from the detector. Figure 2 illustrates the experimental setup. The lens is at position $p$ from the object and the image is at position $p^{\prime}$ from lens. In this case, the object is imaged nearly at the plane of the recording sensor. Let us note $A^{\prime}(x, y)$ the complex field projected onto this plane. In order to optimize the recording, the image of the object must fully lie in the recording plane, so the transverse magnification realized by the lens must be set at $|\gamma|=\min \left(N p_{x} / \Delta A_{x}\right.$; $M p_{y} / \Delta A_{y}$ ), meaning the projected object is fully occupying the horizon of the sensor. In DIPH, the magnification is imposed by the lens whereas in DFH, it can be chosen freely by numerical adjustment of the curvature radius $R_{c}$. In the image-plane configuration, the object wave occupies a spatial frequency bandwidth equal to $(\Delta u \times \Delta v)=\left(\Delta A_{x} / \lambda p \times \Delta A_{y} / \lambda p\right)=\left(|\gamma| \Delta A_{x} / \lambda p^{\prime} \times|\gamma| \Delta A_{y} / \lambda p^{\prime}\right)$. Now, the imagegram is written:

$H=\left|A^{\prime}\right|^{2}+|R|^{2}+A^{\prime} R^{*}+A^{\prime *} R$.

However, the reconstruction distance $d_{r}$ must be at least $d_{r}>\max \left(N p_{x}{ }^{2} / \lambda, M p_{y}{ }^{2} / \lambda\right)$ in order to fulfill the sampling condition of the quadratic phase in the discrete Fresnel transform (equation (7)). This means that, a priori, the imagegram can not be computed by the S-FFT method, whereas it is possible to use it for the Fresnelgram. So, the reconstruction is performed according to the D-FFT strategy with the angular spectrum transfer function (equation (12)), in which $d_{r}=0$ when the object is rigorously projected in the recording plane. Now, the transfer function of the convolution kernel tends to a uniform-bandwidth limited function 
Fig. 2 Optical setup for DIPH and notations
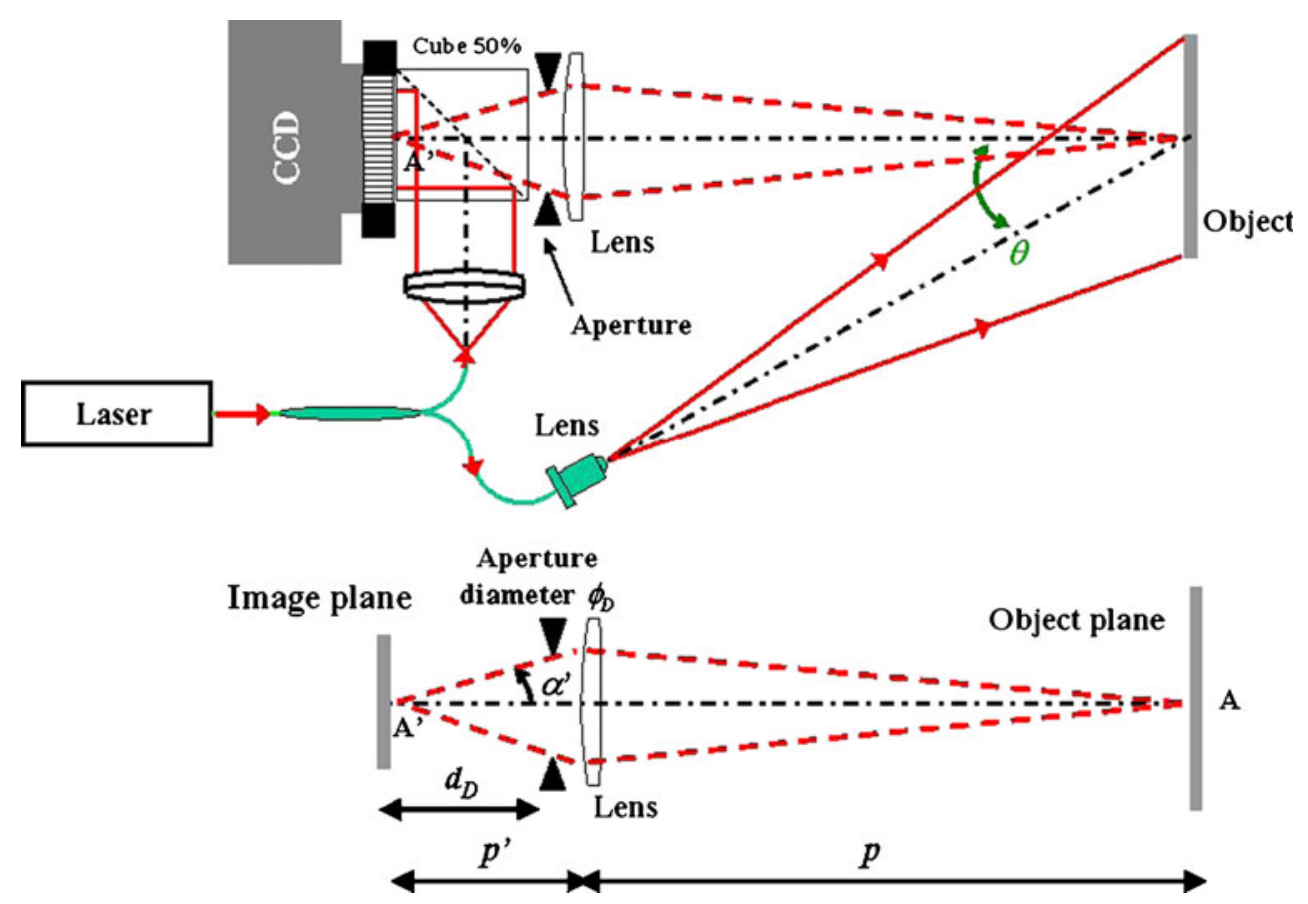

[20, 27-30]. In the Fourier plane, the filtering function can thus be written:

$G^{\prime}(u, v)= \begin{cases}1 & \text { if }\left|u-u_{0}\right| \leq \Delta A_{x} / 2 \lambda p \text { and }\left|v-v_{0}\right| \leq \Delta A_{y} / 2 \lambda p \\ 0 & \text { elsewhere }\end{cases}$

and the object wave is reconstructed according to:

$A_{\mathrm{r}}^{\prime}=F T^{-1}\left[F T[H] \times G^{\prime}\right]$,

Equation (15) is a convolution formula similar to equation (8), but now the impulse response is a two-dimensional sinc function. Note that the transfer function can also be non uniform by choosing an adequate window function (3D Hanning, 2D Tuckey, etc.) in order to reduce the truncation effects.

Influence of the Aperture Diaphragm

This aspect does not clearly appear in literature but it must be pointed out that the imagegram is also the Fresnelgram of the aperture of the imaging system. This means that the DIPH method must be optimized according to the same rules as those for DFH. So, for the Fresnelgram of the aperture, we must apply the same rule as described in equation (5). This means we have:

$d_{D}=\frac{(2+3 \sqrt{2}) p_{x}}{2 \lambda} \phi_{D}$
Hence the numerical aperture of the imaging lens must be set to:

$\sin \alpha^{\prime} \cong \frac{\phi_{\mathrm{D}}}{2 d_{D}}=\frac{\lambda}{(2+3 \sqrt{2}) \mathrm{p}_{\mathrm{x}}}$,

If equation (17) is not fulfilled, the three diffraction orders of the Fresnelgram of the aperture overlap. Thus the useful +1 order of the imagegram (equation (13)) is corrupted by the zero order of the Fresnelgram of the aperture. Note that the numerical aperture of the imaging system only depends on the wavelength and the pixel pitch. It does not depend on the object size, since the optimization of the setup is related to the aperture diameter. In the case of $\mathrm{DFH}$, the useful numerical aperture of the beam is defined according to that of the sensor-to-object beam (Fig. 1). It is equal to $\sin \alpha^{\prime} \cong \Delta A / d_{0}=\lambda /(2+3 \sqrt{ } 2) p_{x}$ thus giving the same result as in equation (17), e.g. about 0.6 deg for a pitch of $8 \mu \mathrm{m}$ and at $532 \mathrm{~nm}$ wavelength. From this standpoint, the optimization of the optical setup follows the same rules for both methods and does not depend on the object size. This has consequences on the spatial resolution of both methods.

The next section discusses some figures of merit so as to objectively compare both methods.

\section{Figures of Merit}

Introduction

This section proposes a theoretical analysis of the influence of the aperture and lens in the case of DIPH. Compared to 
DFH, this element is a critical point that influences several aspects of the reconstruction process: filtering algorithms, spatial resolution and decorrelation noise. A remark concerning the photometric efficiency is also discussed. Moreover, the conditions for optimal filtering and image recovering are established. In order to study the influence of speckle decorrelation, the optical phase from the object reconstruction must be computed. Decorrelation appears when a phase change occurs at the surface of the object. In this paper, we applied a mechanical load to the object with a good reproducibility. By varying the amount of load, different phase changes with different speckle decorrelations are generated. A method based on a low-pass filtering is used to objectively compare the sensitivity of both methods to decorrelation. Equations given for an objective comparison are explained in the sense of the optimal recording, according to the Shannon conditions.

\section{Filtering and Algorithms}

From "Theoretical Basics", we can denote the algorithms have strong similarities. Table 1 gives an overview of the properties of the various reconstruction methods. The simplest reconstruction method is the discrete Fresnel transform used in DFH. The highest complexity is obtained for the convolution method with adjustable magnification but the object can be reconstructed with the same number of data points as the recording sensor [12]. Computation time is given for a PC Pentium 4CPU $2.99 \mathrm{GHz}$ with 2Go RAM equipped with MATLAB 5.3. Note that the computation load is not represented only by the number of FFT calculations, but the latter mainly contributes.
Spatial Resolution

In $\mathrm{DFH}$, the spatial resolution in the reconstructed field is $[11,14]$ :

$\rho_{x}=\frac{\lambda d_{0}}{N p_{x}} \quad \rho_{y}=\frac{\lambda d_{0}}{M p_{y}}$.

The interpretation is rather simple: that is the width of the digital diffraction pattern of a rectangular digital aperture with size $\left(N p_{x} \times M p_{y}\right)$ and uniform transmittance. It depends on the sensor size, wavelength and recording distance. When reconstructing the object by the convolution method with adjustable magnification, the spatial resolution becomes $\rho_{x}^{\gamma}=|\gamma| \rho_{x}$ and $\rho_{y}^{\gamma}=|\gamma| \rho_{y}$ in the reconstructed horizon, which has a size related to $|\gamma|$ [25]. From equations (5) and (18), the equivalent resolution of a Fresnelgram reconstructed in the sensor plane with D-FFT is simply:

$\rho_{x}^{\gamma}=\frac{(2+3 \sqrt{2}) p_{x}}{2} \times \frac{\gamma \Delta A_{x}}{N p_{x}} \quad \rho_{y}^{\gamma}=\frac{(2+3 \sqrt{2}) p_{y}}{2} \times \frac{\gamma \Delta A_{y}}{M p_{y}}$

When the adjustable magnification is set so that the reconstructed object fully lies in the horizon of the sensor (recording horizon), then $\gamma \Delta A_{x} \cong N p_{x}$ (similar relation holds for $y$ direction), leading to:

$\rho_{x}^{\gamma} \cong \frac{(2+3 \sqrt{2}) p_{x}}{2} \quad \rho_{y}^{\gamma} \cong \frac{(2+3 \sqrt{2}) p_{y}}{2}$.

In the case of DIPH, the spatial resolution is influenced by the imaging lens. The impulse response of the full process is related to the impulse response of the imaging lens. Consequently, the spatial resolution is given by the speckle size in the recording plane [31]. The speckle size is

Table 1 Attributes of the reconstruction methods

\begin{tabular}{|c|c|c|c|}
\hline \multirow[t]{2}{*}{ Reconstruction } & \multicolumn{2}{|l|}{$D F H$} & \multirow{2}{*}{$\begin{array}{l}\text { DIPH } \\
\text { Convolution with binary } \\
\text { filtering }\end{array}$} \\
\hline & Discrete Fresnel transform & $\begin{array}{l}\text { Convolution with } \\
\text { magnification }\end{array}$ & \\
\hline Number of FFT operations & 1 & 2 or 3 & 2 \\
\hline $\begin{array}{l}\text { Number of data points for FFT } \\
\text { operations }\end{array}$ & free, $(K, L) \geq(M, N)$ & free, $(K, L) \geq(M, N)$ & free, $(K, L) \geq(M, N)$ \\
\hline $\begin{array}{l}\text { Number of data points for the } \\
\text { reconstructed object }\end{array}$ & imposed, $\Delta A_{x} L p_{x} / \lambda d_{0} \times \Delta A_{y} K p_{y} / \lambda d_{0}$ & free, $\geq M \times N$ & $M \times N$ \\
\hline Filtering & NO & YES & YES \\
\hline Filtering function & - & quadratic phase & binary \\
\hline $\begin{array}{l}\text { Needs for an additional quadratic } \\
\text { phase term }\end{array}$ & NO & YES, for adjustable magnification & NO \\
\hline $\begin{array}{l}\text { Sampling pitches of the reconstructed } \\
\text { object }\end{array}$ & $\left(\lambda d_{0} / L p_{x}, \lambda d_{0} / K p_{y}\right)$ & $\left(p_{x}, p_{y}\right)$ & $\left(p_{x}, p_{y}\right)$ \\
\hline Computation time & $2.703 \mathrm{~s}$ for $(K, L)=(1024,1360)$ & $7.25 \mathrm{~s}$ for $(K, L)=(1024,1360)$ & $3.203 \mathrm{~s}$ for $(K, L)=(1024,1360)$ \\
\hline Complexity & $* *$ & $* * * * *$ & $* * *$ \\
\hline
\end{tabular}


related to the diffraction spot of the aperture of the imaging lens, and is obtained from equation (17):

$\rho_{\mathrm{x}}^{\prime}=\rho_{\mathrm{y}}^{\prime}=\frac{\lambda d_{D}}{\phi_{D}}=\frac{(2+3 \sqrt{2}) p_{x}}{2}$.

Equations (20) and (21) show that, under Shannon conditions, the spatial resolutions are equivalent for both methods. Numerical comparisons of the spatial resolutions are given in "Spatial Resolution".

\section{Influence of Speckle Decorrelation}

A limitation of both methods is given by the speckle decorrelation which occurs when the object is deformed. This decorrelation adds a high spatial frequency noise to the useful signal. Because of this influence, the raw phase maps are not directly suitable for visualization or comparison with some theoretical results. Furthermore, the raw phase map must be unwrapped with a robust noise immune algorithm. Smoothing methods based on sin-cos filtering may be also used [32], resulting in an increase of the signal-to-noise ratio of the phase map. Speckle correlation has been theoretically studied by many authors [32-35]. For studying speckle phase decorrelation, the second-order statistical description is of interest. Especially, the phase decorrelation occurs when comparing two optical phases extracted from recordings. The phase is a random data having the properties of a speckle phase. The reason is that it is closely related to the object surface, which is most often a rough surface. So, description of the correlation property is related to the second-order probability density function of the phase [37, p. 406]. The analytical calculation of the joint probability density function of the phase $\psi_{1}$ and $\psi_{2}$ of two speckle patterns is a difficult one and will not be detailed in this paper. The reader is invited to look at references [37, p. 406] and [38, p. 163]. We note $\varepsilon=\psi_{1}-\psi_{2}$ the noise induced by the speckle decorrelation between two object fields reconstructed after two different states of the object and $\Delta \varphi$ the phase change due to the object loading. Then $\psi_{2}=\psi_{1}+\varepsilon+\Delta \varphi, \Delta \varphi$ being considered as a deterministic variable. The probability density function of $\varepsilon$ depends on the modulus of the complex coherence factor $|\mu|$ between the two speckle fields. With $\beta=|\mu| \cos (\varepsilon)$, the second-order probability density of the phase noise $\varepsilon$ is given by :

$p(\varepsilon)=\frac{1-|\mu|}{2 \pi}\left(1-\beta^{2}\right)^{-3 / 2}\left(\beta \sin ^{-1} \beta+\frac{\pi \beta}{2}+\sqrt{1-\beta^{2}}\right)$.

The signification of equation (22) is the description of the probability for measuring the phase noise $\varepsilon$ in the phase difference between two reconstructions for any loading of the object (mechanic, pneumatic, thermal, acoustic, etc.). Note that in [34], M. Lehmann discussed about the speckle decorrelation in case of resolved and unresolved speckles and by considering smooth-reference-wave and specklereference-wave interferometers. In [34], probability density of the decorrelation induced phase error is derived by taking into account the total number of speckles per pixel, which depends on the ratio between the speckle displacement in the image plane and the pixel size of the sensor. Although equation (22) is derived without taking into account a possible spatial integration due to the pixel surface (resolved or unresolved speckle), it simply depends on a correlation factor $|\mu|$. The plots of equation (22) and of equations given in [34] exhibit the same profiles. It follows that equation (22) can be used as a pertinent indicator so as to compare the decorrelation sensitivity of different experimental methods, the correlation factor $|\mu|$ being a quality marker extracted from experimental data.

The measurement of equation (22) can be performed according to [39]. The subtraction of the low-pass filtered phase difference from the raw phase difference leads to an estimation of the standard deviation of the noise included in the raw data. If $h_{f}(l, k)$ is the $n \times n$ convolution kernel used for the low-pass filtering, then the standard deviation of the measured noise $\sigma_{\Delta}$ is related to the real noise standard deviation $\sigma_{\varepsilon}$ by equation (23):

$\sigma_{\Delta}=\sigma_{\varepsilon} \sqrt{1-2 h_{f}(0,0)+\sum_{k=-n}^{k=+n} \sum_{l=-n}^{l=+n}\left|h_{f}(l, k)\right|^{2}}$.

Equations (22) and (23) are used to analyze the experimental results in "Speckle Decorrelation".

\section{Remark about the Photometric Efficiency}

This subsection discusses about the photometric efficiency of DFH and DIPH. The object is considered to be circular, illuminated by a laser (power $P_{0}$ ), and as a lambertian diffuser with albedo $R_{d}$. We consider $\tau$ the transmission coefficient of the beam splitter cube in front of the sensor. Then, taking into account that $d_{0}$ must be fixed by equation (5) and that the object surface is $S_{O}$, the illumination $I_{D F H}$ given onto the sensor area for the setup of Fig. 1 is given by equation (24):

$I_{D F H}=\frac{\tau R_{d} \lambda^{2} P_{0}}{(2+3 \sqrt{2})^{2} p_{x}^{2} S_{O}}$,

In the case where the object image is projected in the sensor area by the lens, the illumination becomes:

$I_{D I P H}=\frac{\tau R_{d} T_{L} P_{0}}{S_{O}} \sin ^{2} \alpha^{\prime}$, 
where $T_{L}$ is the transmission factor of the imaging lens. Equation (17), equations (24) and (25) lead to the ratio:

$$
\frac{I_{D F H}}{I_{D I P H}}=\frac{1}{T_{L}}>1,
$$

So, the photometric efficiency is slightly in favor of DFH. This result is also independent from the object size, showing that there is no specific advantage for DIPH for large objects compared to DFH. Practically, in the DFH method and from equation (5), a large object must be placed far from the recording area and this could be a limiting aspect. However, it is possible to virtually reduce the object size by using a set of divergent lenses that produces a virtual image smaller and closer from the sensor $[40,41]$. This case leads to $I_{D F H} /$ $I_{D I P H}=1$. But, in terms of photometric efficiency, there is no significant difference between DFH and DIPH (equation (26)).

\section{Experimental Results}

\section{Experimental Parameters}

The holographic set-up is based on a Mach Zehnder configuration (not detailed) in which the sensor has 8 bits digitization with $M \times N=1024 \times 1360$ pixels sized $4.65 \mu \mathrm{m} \times$ $4.65 \mu \mathrm{m}$. The laser is a continuous $\operatorname{HeNe}(\lambda=632.8 \mathrm{~nm}$, $\left.P_{0}=30 \mathrm{~mW}\right)$ and the object is a mechanical structure sized $\Delta A_{x} \times \Delta A_{y}=40 \times 35 \mathrm{~mm}^{2}$. The object is localized at $d_{0}=$ $1030 \mathrm{~mm}$ from the sensor area and is illuminated with a circular spot $40 \mathrm{~mm}$ in diameter. In the case of DIPH, there is an imaging lens associated to a variable aperture close to the lens. The magnification is such that the image of the object entirely covers the sensor area. The spatial frequencies are adjusted according to equation (6). So as to compare both methods, the digital hologram is reconstructed using the discrete Fresnel transform with $(K, L)=(2048,2048)$ and with the convolution method so that $(K, L)=(M, N)=$ $(1024,1360)$ (reconstruction horizon equal to the recording horizon). In the latter case, we get a reconstructed object having the same horizon as the one from DIPH (see Table 1). The magnification for the D-FFT method is almost the same as the physical one obtained with the lens for DIPH, that is $|\gamma| \approx 0.146$ (theoretical value). The lens has a focal length of $150 \mathrm{~mm}$ and the aperture is placed at $145 \mathrm{~mm}$ from the sensor. Equation (16) implies that the optimal diameter in the Shannon sense is $\phi_{D}=6.32 \mathrm{~mm}, \sin \alpha^{\prime}=0.021$, leading to a f\# equal to 23.8. Since the aperture is an iris diaphragm, the diameter is changed with four values $\phi_{D}=\{3.56 ; 5.5 ; 7.41 ; 9.94\} \mathrm{mm}$. Unfortunately, it was not possible to adjust the diaphragm to its optimal diameter. The amplitude of the reference and object beams are adjusted at the same level for both methods. In order to investigate decorrelation, we applied a mechanical loading to the object with an almost good reproducibility. This acceptable reproducibility can be appreciated on experimental results, although the mechanical loadings are not exactly identical. However, this reproducibility is quite sufficient so as to compare the experimental results. For each experimental configuration, we have recorded 5 states of the object corresponding to 4 mechanical loadings. Raw phase maps are filtered by a moving-average filter sized $5 \times 5$ pixels. The moving-average filter used to study the decorrelation noise according to the method described in [39] is sized $n \times n=7 \times 7$ pixels, leading to $\sigma_{\varepsilon}=\sigma_{\Delta} / 0.989$.

\section{Object Reconstructions}

Figure 3 shows the object reconstructed with the three algorithms of Table 1. The exposure time is set to $573 \mathrm{~ms}$. Figure 3(a) shows the full reconstructed field exhibiting the three diffraction orders. The useful part of the field of view corresponding to the reconstructed object is sampled by $511 \times 511$ pixels with pitches $\Delta \eta=\Delta \xi=68.44 \mu \mathrm{m}$. Reconstructions of Fig. 3(b), c are sampled by $M \times N=1024 \times 1360$ pixel with pitches $p_{x}=p_{y}=4.65 \mu \mathrm{m}$.

The image amplitude obtained in Fig. 3(b) is quite similar to that of Fig. 3(c), excepted the small object size difference that can be observed and which is due to the transverse magnification that is not exactly 0.146 for DIPH. Fig. 4
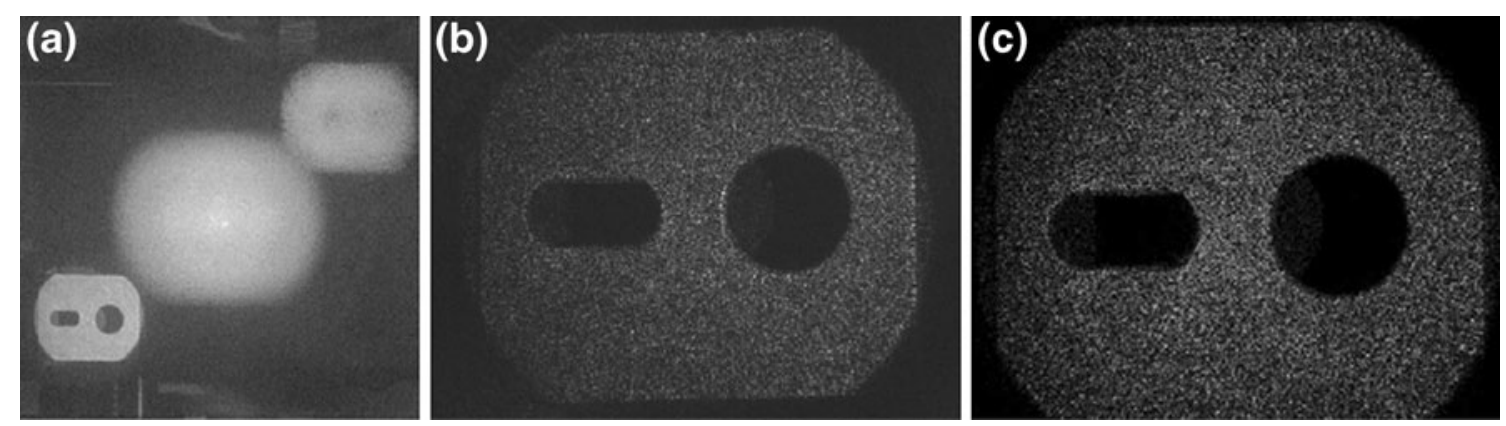

Fig. 3 Object reconstructions, (a) DFH: discrete Fresnel transform, (b) DFH: convolution with adjustable magnification, (c) DIPH 
Fig. 4 Phase differences, (a) DFH: raw with convolution, (b) DFH: filtered, (c) DIPH: raw, (d) DIPH: filtered
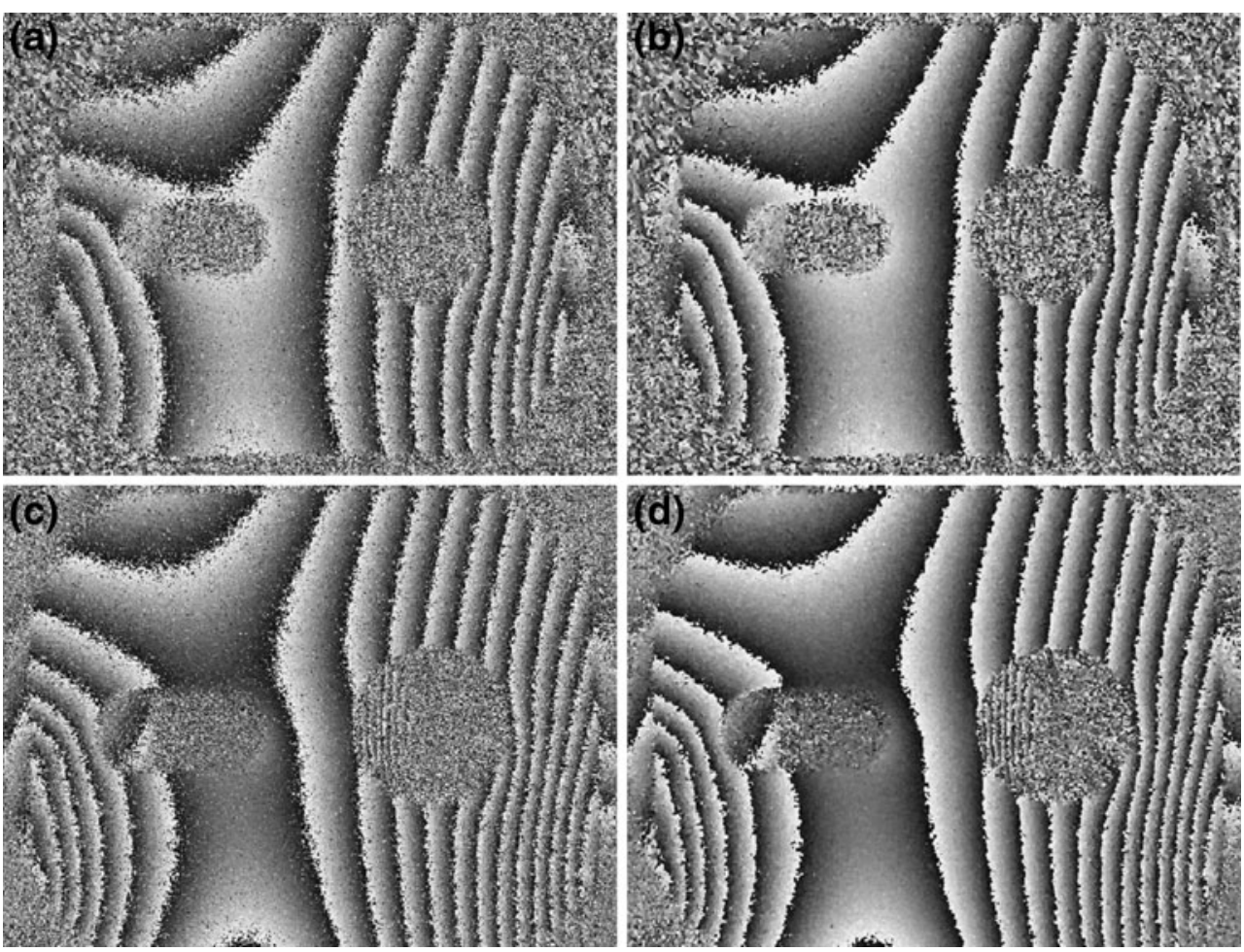

shows phase changes obtained for DFH and DIPH for, approximately, the same loading. Fig. 4(a) and (c) show the raw phase map and the filtered one for DFH. Fig. 4(b) and (d) show the raw phase map and the filtered one for DIPH with $\phi_{D}=5.5 \mathrm{~mm}$. Figure 4 exhibits the very good agreement between both methods and gives appreciation of the acceptable reproducibility of mechanical loading. Indeed, DIPH exhibits only few fringes more than DFH.

\section{Influence of Aperture}

As pointed out, the imagegram is also the Fresnelgram of the aperture. So the aperture can be reconstructed from the Fresnelgram by computing the discrete Fresnel transform with $d_{r}=-d_{D}=-145 \mathrm{~mm}$. When $\phi_{D}>6.3 \mathrm{~mm}$, the diameter does not respect the Shannon conditions, thus the overlapping of the diffraction orders of the aperture occurs. This means that the useful spectral part of the object, also localized at spatial frequencies $\left(u_{0}, v_{0}\right)$ is overlapped by the contribution of the zero-order of the aperture. Consequently, the phase changes between two mechanical loadings are corrupted and the fringe visibility decreases. Figure 5(a, c, e, g) show the reconstructed field with the discrete Fresnel transform and with focus on the iris diaphragm for respectively $\phi_{D}=\{3.56 ; 5.5 ; 7.41 ; 9.94\} \mathrm{mm}$. The image of the aperture can be seen in the bottom left-hand corner of each sub-image. The overlapping can be clearly observed for $\phi_{D} \geq 7.41 \mathrm{~mm}$. Figure $5(b, d, f, h)$ show the phase changes obtained with the four different aperture diameters. The fringe visibility obviously decreases for $\phi_{D} \geq 7.41 \mathrm{~mm}$, the right part of the figure being first affected. Figure 5(h) exhibits the strong visibility decrease obtained for $\phi_{D}=9.94 \mathrm{~mm}$.

This experimental analysis clearly exhibits that the DIPH method is valid only if the numerical aperture of the imaging system is small, typically with a \# greater than 20 .

\section{Spatial Resolution}

From "Spatial Resolution" and experimental parameters, the theoretical spatial resolutions are $\rho_{x}^{\gamma}=15.03 \mu \mathrm{m}$ and $\rho_{y}^{\gamma}=$ $19.85 \mu \mathrm{m}$ for DFH and $\rho_{x}^{\prime}=\rho_{y}^{\prime}=15.51 \mu \mathrm{m}$ for DIPH. These values are close together, which means that the spatial resolutions are the same when fulfilling the Shannon conditions. Experimental measurements are performed as follows: a square zone with $101 \times 101$ pixels is extracted from reconstructed objects and includes sufficient speckle grains. Then the autocorrelation function is computed by FFT algorithms and after normalization $x$-profiles are extracted.

Figure $6(\mathrm{a}, \mathrm{b}, \mathrm{c}, \mathrm{d}, \mathrm{e})$ show the square zones and autocorrelation functions for respectively DFH and DIPH with $\phi_{D}=3.56 \mathrm{~mm}, \phi_{D}=5.5 \mathrm{~mm}, \phi_{D}=7.41 \mathrm{~mm}$ and $\phi_{D}=$ $9.94 \mathrm{~mm} ; x$-profiles of the autocorrelation functions obtained for DFH and indicates the various diameters of the aperture in case of DIPH are also presented. Figure 6 shows that spatial resolutions are comparable for all cases. It is slightly better for $\phi_{D}=7.41 \mathrm{~mm}$ since the autocorrelation is narrower, but for $\phi_{D}=9.94 \mathrm{~mm}$, the resolution is degraded since the curve is wider than the theoretical speckle size 

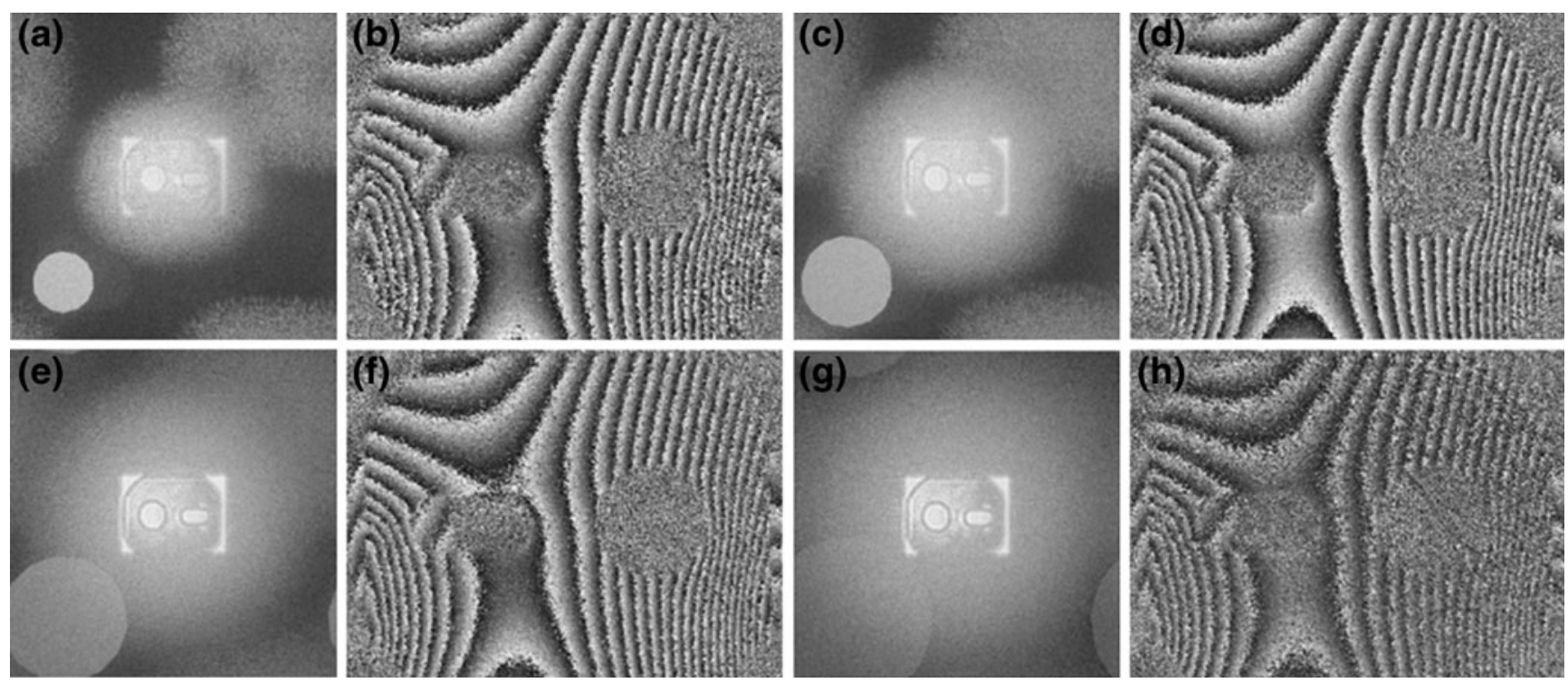

Fig. 5 Holographic reconstructions of the aperture (a) $\phi_{D}=3.56 \mathrm{~mm},(\mathbf{c}) \phi_{D}=5.5 \mathrm{~mm}$, (e) $\phi_{D}=7.41 \mathrm{~mm},(\mathbf{g}) \phi_{D}=9.94 \mathrm{~mm}$ and phase differences obtained with SI (b) $\phi_{D}=3.56 \mathrm{~mm},(\mathbf{d}) \phi_{D}=5.5 \mathrm{~mm}$, (f) $\phi_{D}=7.41 \mathrm{~mm},(\mathbf{g}) \phi_{D}=9.94 \mathrm{~mm}$

$(9.23 \mu \mathrm{m})$. So, it appears that the increase of the aperture does not enhance the resolution as the curve for $\phi_{D}=$ $9.94 \mathrm{~mm}$ is wider than for $\phi_{D}=3.56 \mathrm{~mm}$. The reason is that the overlapping of the aperture in the Fresnelgram contributes to degrade the spatial resolution when the aperture diaphragm has a diameter exceeding a certain value. So there is no gain to increase the diameter of the aperture diaphragm.

Speckle Decorrelation

As discussed previously, the influence of the speckle decorrelation is estimated for the measurement of mechanical deformations. Therefore, we have applied almost the same mechanical loading in both experimental configurations and then estimated the probability density of the noise maps. We applied four mechanical loadings increased in constant steps. Fitting the curve according to equation (22) results in an objective comparison of the decorrelation degrees, and this gives keys to compare the decorrelation sensitivity of the methods. Figure 7 shows the probability density function of speckle decorrelation for both DFH and DIPH and for two states of deformations (1) and (2). State (1) corresponds to the deformation between the $3 \mathrm{rd}$ and the $1 \mathrm{st}$ recording and state (2) corresponds to the deformation between the 5 th and the 1 st recording. The estimated value of $|\mu|$ and the noise standard deviation $\sigma_{\varepsilon}$ are indicated for each curve. Table 2 presents the experimental values of $|\mu|$ and $\sigma_{\varepsilon}$ for both methods, depending on the diameter of the aperture, and for the two states of loading. Figure 7 shows that the speckle decorrelation is strongly influenced by the aperture
Fig. 6 [COLOR ONLINE]. Speckle grains and autocorrelation functions for (a) DFH and DIPH, (b) $\phi_{D}=3.56 \mathrm{~mm},(\mathbf{c})$ $\phi_{D}=5.5 \mathrm{~mm}$, (d) $\phi_{D}=7.41 \mathrm{~mm}$ (e) $\phi_{D}=9.94 \mathrm{~mm}$; left: $x$ profiles of autocorrelation functions.
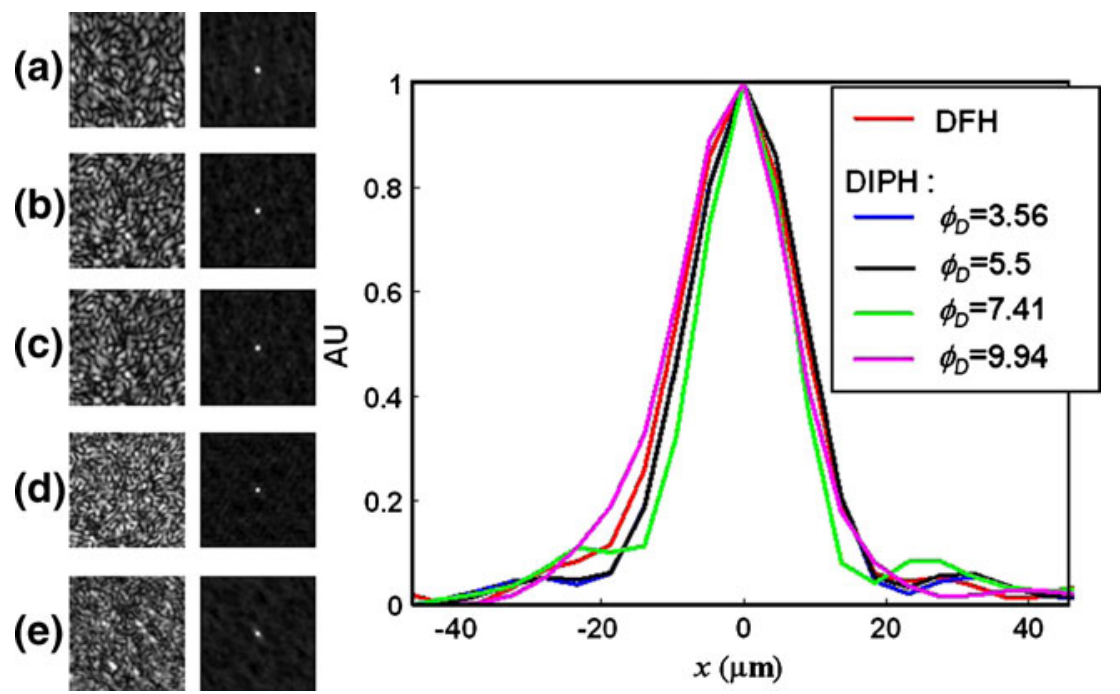
Fig. 7 [COLOR ONLINE]. Sensitivity to decorrelation, (1) deformation between the 3rd and the 1st recording, (2) between the 5 th and the 1 st recording, - experimental data, o fitting with equation (22)
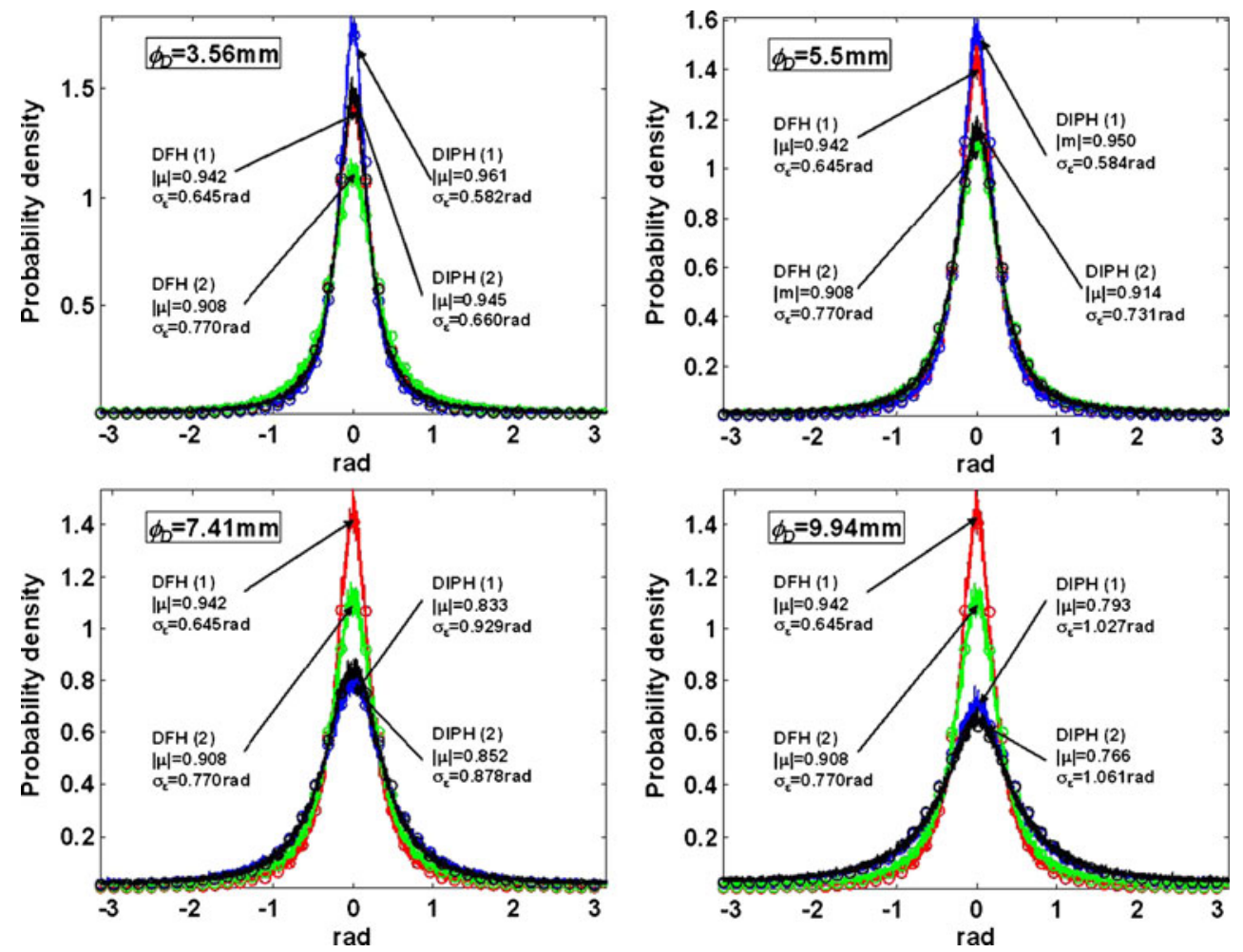

size. It shows that the speckle decorrelation increases with the increase of the aperture diaphragm $\left(\phi_{D}=9.94 \mathrm{~mm}\right)$, and seems to be "saturated" since there is no significant difference between the two states of loadings, whereas it is not the case for $\phi_{D}=3.56 \mathrm{~mm}$ (see Table 2). Consequently, DFH is less sensitive to decorrelation than DIPH, if the numerical aperture is greater than $\lambda /(2+3 \sqrt{2}) p_{x}$ (equation (17) not fulfilled). This statement that DIPH is more sensitive to decorrelation at larger apertures disagrees with the well known results of Lehmann [34-36], who demonstrated that the speckle decorrelation decreases with the increase of the diameter aperture diameter. This contradiction is due to the influence of the 0 order of the Fresnelgram of the aperture that induces more phase fluctuations than the one produced by the pure speckle decorrelation induced by the mechanical loading. In the case of $\sin \alpha^{\prime}<\lambda /(2+3 \sqrt{2}) p_{x}$, DIPH is less sensitive than DFH. When the Shannon conditions are fulfilled, $\sin \alpha^{\prime}=\lambda /$

Table 2 Measurement of speckle decorrelation

\begin{tabular}{llllll}
\hline Method & \multicolumn{2}{l}{ Load (1) } & & \multicolumn{2}{l}{ Load (2) } \\
\cline { 2 - 3 } \cline { 5 - 6 } & $|\mu|$ & $\sigma_{\varepsilon(\mathrm{rad})}$ & & $|\mu|$ & $\sigma_{\varepsilon}(\mathrm{rad})$ \\
\hline DFH & 0.942 & 0.645 & 0.908 & 0.770 \\
DIPH: $\phi_{D}=3.56 \mathrm{~mm}$ & 0.961 & 0.582 & 0.945 & 0.660 \\
DIPH: $\phi_{D}=5.5 \mathrm{~mm}$ & 0.950 & 0.584 & 0.914 & 0.731 \\
DIPH: $\phi_{D}=7.41 \mathrm{~mm}$ & 0.833 & 0.929 & & 0.852 & 0.878 \\
DIPH: $\phi_{D}=9.94 \mathrm{~mm}$ & 0.793 & 1.027 & 0.766 & 1.061 \\
\hline
\end{tabular}

$(2+3 \sqrt{2}) p_{x}$, both methods have the same sensitivity to speckle decorrelation. This can be appreciated in Fig. 7 with $\phi_{D}=5.5 \mathrm{~mm}$ for which both DFH and DIPH curves are quasi superposed (see also Table 2). For $\phi_{D}=6.3 \mathrm{~mm}$ (Shannon conditions for the aperture) the curves may be expected to be perfectly superposed.

\section{Conclusion}

This paper exposes some figures of merit so as to compare digital Fresnel holography (DFH) and digital image-plane holography (DIPH). Because of the simplicity of the Fresnel transform, digital Fresnel holography is quite more adapted to simple and automated image processing. The role of the aperture diaphragm in the digital image-plane holography configuration is highlighted. It is shown that the aperture diaphragm must fulfill the Shannon condition of its own Fresnelgram. In DIPH, if the sampling conditions are not fulfilled, the reconstructed object and phase is corrupted by a noise due to the overlapping of the diffraction order of the aperture. So, the spatial resolutions of DFH and DIPH are almost the same. The sensitivity to speckle decorrelation is increased with the increase of the aperture. This result is amazing considering previous studies on speckle decorrelation, but it can be explained by the primordial influence of the aperture diaphragm as zero-order overlapping induces a strong decrease of the signal-to-noise ratio, both in the object amplitude and phase. As shown in the paper, both 
methods lead to good displacement maps. The main advantage for DFH is simple computing while as DIPH uses the lens to image the object it is then possible to shorten the object arm.

Acknowledgments The authors are grateful to Pierre Jacquot (EPFL, Lausanne, Switzerland) for very helpful discussions.

\section{References}

1. Schnars U, Jüptner W (1994) Direct recording of holograms by a CCD target and numerical reconstruction. Appl Opt 33:179-181

2. Yamaguchi I, Zhang T (1997) Phase shifting digital holography. Opt Lett 22:1268-1270

3. Yamaguchi I, Kato J, Ohta S, Mizuno J (2001) Image formation in phase shifting digital holography and application to microscopy. Appl Opt 40:6177-6186

4. Cuche E, Bevilacqua F, Depeursinge C (1999) Digital holography for quantitative phase contrast imaging. Opt Lett 24:291-293

5. De Nicola S, Ferraro P, Finizio A, Pierattin G (2001) Correctimage reconstruction in the presence of severe anamorphism by mean of digital holography. Opt Lett 26:974-976

6. Picart P, Diouf B, Lolive E, Berthelot J-M (2004) Investigation of fracture mechanisms in resin concrete using spatially multiplexed digital Fresnel holograms. Opt Eng 43:1169-1176

7. Yamaguchi I, Matsumura T, Kato J (2002) Phase shifting color digital holography. Opt Lett 27:1108-1110

8. Tankam P, Song Q, Karray M, Li JC, Desse JM, Picart P (2010) Real-time three-sensitivity measurements based on three-color digital Fresnel holographic interferometry. Opt Lett 35:2055-2057

9. Picart P, Leval J, Mounier D, Gougeon S (2003) Time-averaged digital holography. Opt Lett 28:1900-1902

10. Onural L (1993) Diffraction from a wavelet point of view. Opt Lett $18: 846-848$

11. Kreis Th, Adams M, Jüptner W (1997) Methods of digital holography: a comparison. Proc SPIE 3098:224-233

12. Li JC, Tankam P, Peng Z, Picart P (2009) Digital holographic reconstruction of large objects using a convolution approach and adjustable magnification. Opt Lett 34:572-574

13. Wagner C, Seebacher S, Osten W, Jüptner W (1999) Digital recording and numerical reconstruction of lens less Fourier holograms in optical metrology. App Opt 38:4812-4820

14. Picart P, Leval J (2008) General theoretical formulation of image formation in digital Fresnel holography. J Opt Soc Am A 25:17441761

15. Creath K (1985) Phase shifting speckle interferometry. Appl Opt 24:3053-3058

16. Kreis Th (1996) Holographic Interferometry - Principles and Methods Akademie Verlag Series in Optical Metrology 1. Akademie Verlag Gmbh, Berlin

17. Picart P, Leval J, Piquet F, Boileau J-P, Guimezanes Th, Dalmont J-P (2007) Tracking high amplitude autooscillations with digital Fresnel holograms. Optic Express 15:8263-8274

18. Aguayo D, Mendoza Santoyo F, De la Torre-Ibarra MH, SalasAraiza MD, Caloca-Mendez C, Gutierrez Hernandez DA (2010) Insect wing deformation measurements using high speed digital holographic interferometry. Optic Express 18:5661-5667
19. Pérez-López C, De la Torre-Ibarra MH, Mendoza Santoyo F (2006) Very high speed cw digital holographic interferometry. Optic Express 14:9709-9715

20. Pedrini G, Tiziani H, Zou Y (1997) Digital double pulse-TV holography. Opt \& Las Eng 26:199-219

21. Doval ÁF, Trillo C (2006) Hybrid opto-numerical quasi Fourier transform digital holographic camera. Proc SPIE 6341:63410Z

22. Jacquot $P$ (2008) Speckle interferometry: A review of the principal methods in use for experimental mechanics applications. Strain 44:57-69

23. Goodman JW (1996) Introduction to fourier optics, 2nd edn. McGraw-Hill, New York

24. Picart P, Tankam P, Mounier D, Peng Z, Li JC (2009) Spatial bandwidth extended reconstruction for digital color Fresnel holograms. Optic Express 17:9145-9156

25. Tankam P, Picart P, Mounier D, Desse JM, Li JC (2010) Method of digital holographic recording and reconstruction using a stacked color image sensor. Appl Opt 49:320-328

26. Zhang F, Yamaguchi I, Yaroslavsky LP (2004) Algorithm for reconstruction of digital holograms with adjustable magnification. Opt Lett 29:1668-1670

27. Saldner HO, Molin NE, Stetson KA (1996) Fourier-transform evaluation of phase data in spatially phase-biased TV holograms. Appl Opt 35:332-336

28. Schedin S, Pedrini G, Tiziani H, Santoyo FM (1999) Simultaneous three-dimensional dynamic deformation measurements with pulsed digital holography. Appl Opt 38:7056-7062

29. Pedrini G, Tiziani H (1997) Quantitative evaluation of twodimensional dynamic deformations using digital holography. Opt Las Technol 29:249-256

30. Pedrini G, Froening Ph, Fessler H, Tiziani H (1997) Transient vibration measurements using multipulse digital holography. Opt Las Technol 29:505-511

31. Dainty JC (1984) Laser speckle and related phenomena. Springer Verlag, Berlin

32. Aebischer HA, Waldner S (1999) A simple and effective method for filtering speckle-interferometric phase fringe patterns. Opt Comm 162:205-210

33. Owner-Petersen P (1991) Decorrelation and fringe visibility: on the limiting behavior of various electronic speckle-pattern correlation interferometers. J Opt Soc Am A 8:1082-1089

34. Lehmann M (1997) Decorrelation-induced phase errors in Phase Shifting Speckle Interferometry. Appl Opt 36:3657-3667

35. Lehmann M (1996) Phase-shifting speckle interferometry with unresolved speckles: A theoretical investigation. Opt Comm 128:325-340

36. Lehmann M (1995) Optimization of wave-field intensities in phase-shifting speckle interferometry. Opt Comm 118:199-206

37. Middleton D (1960) Introduction to statistical communication theory. Mc Graw Hill, New York

38. Davenport WB, Root WL (1958) Random signals and noise. Mc Graw Hill, New York

39. Picart P, Mercier R, Lamare M, Breteau J-M (2001) A simple method for measuring the random variation of an interferometer. Meas Sci Technol 12:1311-1317

40. Schnars U, Kreis TM, Juptner WPO (1996) Digital recording and numerical reconstruction of holograms: Reduction of the spatial frequency spectrum. Opt Eng 35:977-982

41. Mundt J, Kreis TM (2010) Digital holographic recording and reconstruction of large scale objects for metrology and display. Opt Eng 49:125801-1-6 